\title{
Supersymmetry approach to the random heteropolymer theory
}

\author{
Alexander I. Olemskoi \\ Physical Electronics Department, Sumy State University \\ 2, Rimskii-Korsakov Str., 244007 Sumy UKRAINE \\ E-mail: Alexander@olem.sumy.ua
}

\begin{abstract}
The effective equation of motion that describes the different monomer alternation along the heteropolymer chain is proposed. On this basis the supersymmetry field scheme is built up to analyze memory and ergodicity breaking effects.
\end{abstract}

PACS numbers: 61.41.+e, 05.40+j, 11.30.Pb 
Heteropolymer represents an ensemble of chains of different type monomers (many-letter sequences), that can experience the freezing and the microphase separation (MS) (see [1] and references therein). Firstly, MS was studied within random phase approximation [2] for the case of block copolymer $\mathrm{AB}$ with arbitrary fraction $f$ of monomers of type A. At $f \neq 0.5$ with temperature decrease the system undergoes MS sequence of the first order, where period $2 \pi / k_{0}$ of space structure is of order of block length and does not depend on temperature. By virtue of condition $k_{0} \neq 0$ the order parameter fluctuations give divergent contribution to thermodynamic values, so that continuous phase transition transforms to the weak first order transition [3]. Passing to random heteropolymers both MS and freezing transitions remain, but here the space period is strongly dependent on temperature [1, 4]. According to the field considerations [5, 6] the fluctuations suppress both transitions in random copolymers, but copolymer melts in disordered media suffer these transformations.

Similar to spin glass [7], the main tool of statistical mechanics of random heteropolymers is the replica trick. Besides, the transfer matrix method [8] and kinetic approach [9] have been used. At the same time, it is well known in theory of spin glass that within the framework of Sherrington-Kirkpatrick model the replica method is identical to the supersymmetry (SUSY) field approach [10,11]. Being based on introducing Grassmann variables, the SUSY field components have explicit physical meaning, so that in contrast to the replica trick the SUSY method arrives at physically interpreted results [12]. Up to now, the SUSY method in polymer theory has been proposed by Vilgis [13] and did not obtain further development.

Usually, the SUSY method is based on the equation of motion of Langevin type. But covalent bonds, that ensure the formation of polymer chain, make such way ineffective because the dynamic theory of polymers is much more complicated than statistical mechanics of usual many-body systems [14]. Therefore, one needs the effective motion equation instead of dynamical one. The main aim of this work is to obtain such equation for random heteropolymers and to build up corresponding statistical SUSY scheme.

Let us start with the directed polymer that represents Gaussian chain with the probability $\Psi(\mathbf{R}, N)$ to find the end-point $N$ at the point $\mathbf{R}$. As is known, the function $\Psi(\mathbf{R}, N)$ obeys the Schroedinger-type equation with imaginary time -iN [14]

$$
\partial \Psi / \partial N=\left(D \partial^{2} / \partial \mathbf{R}^{2}-U(\mathbf{R}, N)\right) \Psi
$$

where number $N \gg 1, D$ is the effective diffusion coefficient, $U(\mathbf{R}, N)$ is the external field. In the limit where $N \rightarrow \infty$ the solution of Eq.(1) can be written in the form of functional integral over dependence $\mathbf{r}(n)$ of chain coordinate on number of internal monomers:

$$
\Psi(\mathbf{R}, N)=\int \exp \left(-S_{\mathbf{R} N}\{\mathbf{r}(n)\} / 2 D\right) \delta \mathbf{r}(n)
$$

Here the action $S(\mathbf{R}, N) \equiv S_{\mathbf{R} N}\{\mathbf{r}(n)\}=\int_{0}^{N} L_{0}(\mathbf{r}(n)) \mathrm{d} n$ with the fixed end-points $\mathbf{r}(0)=\mathbf{0}$ and $\mathbf{r}(N)=\mathbf{R}$ is determined by Lagrangian of the Euclidean field theory [10]

$$
L_{0}=\frac{1}{2}\left(\frac{\mathrm{d} \mathbf{r}(n)}{\mathrm{d} n}\right)^{2}+2 D U(\mathbf{r}, n),
$$

where the effective kinetic energy, for which the continuum limit $\mathbf{r}(n+1)-\mathbf{r}(n) \rightarrow \mathrm{d} \mathbf{r}(n) / \mathrm{d} n$ is assumed, is caused by the covalent bond between monomers of polymer chain [14]. Inserting 
Eq.(2) to Eq.(1) gives the Jacobi-type equation $\partial S / \partial N=D \partial^{2} S / \partial \mathbf{R}^{2}-(1 / 2)(\partial S / \partial \mathbf{R})^{2}+$ $2 D U$. After introducing the generalized momentum $\mathbf{p} \equiv \partial S / \partial \mathbf{R}$ and the total derivative $\mathrm{d} \mathbf{p} / \mathrm{d} N \equiv \partial \mathbf{p} / \partial N+(\mathbf{p} \partial / \partial \mathbf{R}) \mathbf{p}$ the last equation takes the linear form by Burgers: $\mathrm{d} \mathbf{p} / \mathrm{d} N=D\left(\partial^{2} \mathbf{p} / \partial \mathbf{R}^{2}+2 \partial U / \partial \mathbf{R}\right)$. The above relations are well-known in theories of directed polymers, stochastic growth and kinetic roughening phenomena (see [15]).

Our basic observation [16] is that the Schroedinger-type equation (11) takes the form of the Fokker-Planck equation

$$
\frac{\partial P}{\partial N}=\left(D \frac{\partial^{2}}{\partial \mathbf{R}^{2}}-\frac{\partial}{\partial \mathbf{R}} \mathbf{F}\right) P
$$

for the probability distribution

$$
P(\mathbf{R}, N)=\Psi(\mathbf{R}, N) \exp \{-V(\mathbf{R}) / 2 D\},
$$

where the dependence on $\mathbf{R}$ is determined by the effective potential $V \equiv-\int \mathbf{F d} \mathbf{R}$. Corresponding force $\mathbf{F}$ is related to the initial potential $U$ in Eq.(1]) as follows: $U=$ $\mathbf{F}^{2} / 4 D+(1 / 2) \partial \mathbf{F} / \partial \mathbf{R}$. As is well known [17], Eq.(四) determines the probability to realize solution of corresponding Langevin-type equation

$$
\partial \mathbf{R} / \partial N=\mathbf{F}(\mathbf{R}, N)+\zeta(N)
$$

for stochastic function $\mathbf{R}=\mathbf{R}(N)$. Here the stochastic force $\zeta$ is subjected to the white-noise conditions $\langle\zeta(N)\rangle=0,\left\langle\zeta(N) \zeta\left(N^{\prime}\right)\right\rangle=2 D \delta\left(N-N^{\prime}\right)$, where the angular brackets denote averaging with respect to the distribution (5).

In going from the case of directed polymer to the random heteropolymer $\mathrm{AB}$, the coordinate $\mathbf{R}$ of the end-point $N$ becomes a stochastic Ising variable $\theta(n)$, where $\theta(n)=1$ if $n$-th segment is of type $\mathrm{A}$ and $\theta(n)=-1$ otherwise. Corresponding to the quenched disorder in fixed sequence of different type segments, the law $\theta(n)$ of monomer alternation along the chain is described by the master equation analogous to that determining the Glauber dynamics [18]. Relevant sequence correlator $\overline{\sigma(n) \sigma\left(n^{\prime}\right)}$ for effective spin $\sigma(n) \equiv \theta(n)-\overline{\theta(n)}$, being deviation of microscopic value $\theta(n)$ from the average $\overline{\theta(n)}$, takes the form [8]

$$
\begin{aligned}
& \overline{\sigma(n) \sigma\left(n^{\prime}\right)}=C_{2} \exp \left(-\left|n-n^{\prime}\right| / l\right), \\
& C_{2} \equiv 4 f(1-f), \quad f \equiv(1 / 2)(1+\overline{\theta(n)}),
\end{aligned}
$$

where overbar denotes the averaging over composition sequence (quenched disorder), $l$ is the correlation length and $f$ is the fraction of type-A monomers.

Possessing the exponential correlator (7), stochastic variable $\sigma(n)$ is governed by the effective motion equation $\mathrm{d} \sigma / \mathrm{d} n=-\sigma / l+s(n)$ with the white noise: $\overline{s(n)}=0, \overline{s(n) s\left(n^{\prime}\right)}=$ $2 C_{2} l^{-1} \delta\left(n-n^{\prime}\right)$. Relation between the microscopic value $\sigma(n)$ and stochastic $\delta$-correlated variable $s(n)$ is given by the equality [17] $\sigma(n)=\int_{0}^{n} e^{-(n-m) / l} s(m) \mathrm{d} m$. Contrary to the colored noise $\sigma(n)$, the white noise $s(n)$ has the Gaussian distribution function determining quenched disorder with the intensity $C_{2} l^{-1}$. Then the locally averaging field

$$
\eta(\mathbf{r}, n) \equiv\left(4 C_{2}\right)^{-1 / 2} \overline{\sigma(n) \delta(\mathbf{r}-\mathbf{r}(n))}
$$

represents the order parameter (hereafter the monomer volume is assumed to be equal unity). 
In addition to the terms of type included in in Eq.(6), the effective equation of motion for field (8) must contain the inhomogeneity contribution $D \partial^{2} \eta / \partial \mathbf{r}^{2}$ that has Fourier transform $-D k^{2} \eta_{\mathbf{k}}$. As a result, the equation of motion for the Fourier transformation $\eta_{\mathbf{k}}(n)$ of field (8) takes the Langevin form:

$$
\partial \eta_{\mathbf{k}} / \partial n=-D k^{2} \eta_{\mathbf{k}}-\partial \mathcal{H} / \partial \eta_{\mathbf{k}}^{*}+\zeta_{\mathbf{k}} .
$$

Here, as above, the continuum approximation is used for effective time $n \gg 1$, and the definition of effective force $f_{\mathbf{k}}=-\partial \mathcal{H} / \partial \eta_{\mathbf{k}}^{*}$ is taken into account. The white noise $\zeta_{\mathbf{k}}=\zeta_{\mathbf{k}}(n)$ is defined by the conditions

$$
\left\langle\zeta_{\mathbf{k}}\right\rangle=0, \quad\left\langle\zeta_{\mathbf{k}}^{*}(n) \zeta_{\mathbf{k}^{\prime}}\left(n^{\prime}\right)\right\rangle=2 \delta_{\mathbf{k k}^{\prime}} \delta\left(n-n^{\prime}\right),
$$

where the angular brackets denote averaging over chain conformations (thermal disorder). Taking into account the effect of fluctuations, the effective Hamiltonian reads [1:5:8]

$$
\begin{aligned}
& \mathcal{H}=\sum_{\mathbf{k}} r_{\mathbf{k}}\left|\eta_{\mathbf{k}}\right|^{2}-\frac{1}{2} \sum_{\mathbf{k k}^{\prime}} w_{\mathbf{k k}^{\prime}}\left|\eta_{\mathbf{k}}\right|^{2}\left|\eta_{\mathbf{k}^{\prime}}\right|^{2}+\int v(\mathbf{r}) \mathrm{d} \mathbf{r} ; \\
& r_{\mathbf{k}} \equiv r+2 D\left(k-k_{0}\right)^{2}, \quad k_{0}^{-1} \equiv 2(\pi D)^{1 / 2} l(2 r)^{1 / 4}, \\
& r=\tau+(3 / 4 \pi) l^{-2}(2 r)^{-1 / 2}, \quad \tau \equiv l^{-1}-C_{2} \chi \\
& w_{\mathbf{k k}^{\prime}} \equiv 4 \sigma^{2} l^{-2}(N D)^{-1}\left(\mathbf{k}^{2}+\mathbf{k}^{\prime 2}\right)^{-1} \\
& v=-(\mu / 3 !) \eta^{3}+(\lambda / 4 !) \eta^{4}, \\
& \mu \equiv 12 C_{3} C_{2}^{-1 / 2} l^{-1}, \quad \lambda \equiv 24\left(1+5 C_{3}^{2} / C_{2}\right) l^{-1} \\
& C_{2} \equiv 4 f(1-f), \quad C_{3} \equiv|1-2 f| .
\end{aligned}
$$

Here $\sigma, \chi$ are the interreplica overlapping and the Flory parameters, correspondingly.

The following application of the SUSY scheme is straightforward [10]. One has to introduce the generating functional

$$
\begin{aligned}
& Z\left\{\eta_{\mathbf{k}}\right\}=\left\langle\delta\left(\frac{\partial \eta_{\mathbf{k}}}{\partial n}+\frac{\delta \mathcal{H}}{\delta \eta_{\mathbf{k}}^{*}}-\zeta_{\mathbf{k}}\right) \operatorname{det}\left|\frac{\delta \zeta_{\mathbf{k}}}{\delta \eta_{\mathbf{k}}}\right|\right\rangle, \\
& \delta \mathcal{H} / \delta \eta_{\mathbf{k}}^{*} \equiv \partial \mathcal{H} / \partial \eta_{\mathbf{k}}^{*}+2 D\left(k-k_{0}\right)^{2} \eta_{\mathbf{k}},
\end{aligned}
$$

being the average over noise $\zeta_{\mathbf{k}}(n)$, where $\delta$-function accounts for the motion equation (9), the determinant is Jacobian of transformation from $\zeta_{\mathbf{k}}$ to $\eta_{\mathbf{k}}$. Then the functional Laplace representation is used for $\delta$-function, that gives a ghost field $\varphi_{\mathbf{k}}(n)$. One needs to use Grassmann conjugated fields $\psi_{\mathbf{k}}(n), \bar{\psi}_{\mathbf{k}}(n)$ [10] in order to write the determinant in exponential form. Then, assuming that conformation averaging in Eq.(12) is Gaussian with variance 1 (see Eqs.(10)), the standard form is derived (cf. Eqs. $(2,3)$ )

$$
\begin{aligned}
& Z\{\eta\}=\int P\{\eta, \varphi ; \psi, \bar{\psi}\} \delta \varphi \delta^{2} \psi ; \quad \delta^{2} \psi \equiv \delta \psi \delta \bar{\psi}, \\
& P\{\eta, \varphi ; \psi, \bar{\psi}\}=\exp (-S\{\eta, \varphi ; \psi, \bar{\psi}\}), \quad S=\int_{0}^{N} L \mathrm{~d} n, \\
& L=\int\left[\left(\varphi \dot{\eta}+\bar{\psi} \dot{\psi}-\varphi^{2} / 2\right)+\left(\mathcal{H}^{\prime}\{\eta\} \varphi+\bar{\psi} \mathcal{H}^{\prime \prime}\{\eta\} \psi\right)\right] \mathrm{d} \mathbf{r} .
\end{aligned}
$$


Here, the point denotes the derivative with respect to "time" $n$, the prime denotes the functional derivative with respect to the field (8).

The last expression in Eqs.(13) takes the simplest form

$$
\begin{aligned}
L & =\frac{1}{2} \int \Lambda(\Phi) \mathrm{d}^{2} \vartheta, & \Lambda \equiv \sum_{\mathbf{k}} \Phi_{\mathbf{k}}^{*} \overline{\mathcal{D}} \mathcal{D} \Phi_{\mathbf{k}}+\mathcal{H}\left\{\Phi_{\mathbf{k}}\right\} \\
\mathcal{D} & \equiv \partial / \partial \bar{\vartheta}+\vartheta \partial / \partial n, & \overline{\mathcal{D}} \equiv \partial / \partial \vartheta+\bar{\vartheta} \partial / \partial n
\end{aligned}
$$

within the SUSY field representation

$$
\Phi=\eta+\bar{\psi} \vartheta+\bar{\vartheta} \psi+\bar{\vartheta} \vartheta \phi
$$

where Grassmann coordinates $\vartheta, \bar{\vartheta}$ obey the same relations as fields $\psi, \bar{\psi}$. Here the new field $\phi_{\mathbf{k}} \equiv \dot{\eta}_{\mathbf{k}}-\varphi_{\mathbf{k}}$ is introduced, the functional $\mathcal{H}\{\Phi\}$ has the same form as the effective Hamiltonian (11), where the order parameter $\eta_{\mathbf{k}}$ is replaced by the SUSY field $\Phi_{\mathbf{k}}$, Eq.(15), and the term $\left(\delta \mathcal{H} / \delta \eta_{k}^{*}\right) \eta_{\mathbf{k}}+$ c.c. is the total derivative $\mathrm{d} \mathcal{H}\left\{\eta_{\mathbf{k}}(n)\right\} / \mathrm{d} n$ that can be omitted. According to [12], the physical meaning of the SUSY field components is as follows: $\phi \equiv$ $-\delta \mathcal{H} / \delta \eta^{*}$ is the field being conjugated to the order parameter $\eta, \varphi \equiv \dot{\eta}-\phi$ is the most probable value of fluctuation of the conjugate field, and the combination $\psi \bar{\psi}$ gives the density of sharp interphases. So, the using of the whole 4-component SUSY field (15) corresponds to the strong segregation limit. In what follows we concentrate on the simple case of the weak segregation limit where $\psi \bar{\psi} \equiv 0$. Then, the SUSY field (15) is reduced to the $2-$ component form

$$
\Phi=\eta+\theta \varphi
$$

where self-conjugated nilpotent variable $\theta \equiv \bar{\vartheta} \vartheta$ is introduced. As a result, the Lagrangian (114) takes the form

$$
\begin{aligned}
L & =\frac{1}{2} \int \Lambda(\Phi) \mathrm{d} \theta, \quad \Lambda \equiv \sum_{\mathbf{k}} \Phi_{\mathbf{k}}^{*} D \Phi_{\mathbf{k}}+\mathcal{H}\left\{\Phi_{\mathbf{k}}\right\} \\
D & =-\partial / \partial \theta+(1-2 \theta \partial / \partial \theta) \partial / \partial n .
\end{aligned}
$$

Here the fluctuation field $\varphi$ is used instead of conjugate field $\phi$ and the lowest power of derivative $\partial / \partial n$ is retained. To correspond to Lagrangian (17) the motion equation for SUSY field (16) reads

$$
D \Phi_{\mathbf{k}}=-\delta \mathcal{H} / \delta \Phi_{\mathbf{k}}^{*}
$$

Let us introduce now the SUSY correlator

$$
C_{\mathbf{k}}\left(n, \theta ; n^{\prime}, \theta^{\prime}\right) \equiv\left\langle\Phi_{\mathbf{k}}^{*}(n, \theta) \Phi_{\mathbf{k}}\left(n^{\prime}, \theta^{\prime}\right)\right\rangle \vartheta\left(n-n^{\prime}\right),
$$

where $\vartheta\left(n-n^{\prime}\right)=1$ if $n>n^{\prime}$ and $\vartheta\left(n-n^{\prime}\right)=0$ otherwise. Multiplying Eq.(18) by $\Phi_{\mathbf{k}}^{*}$ and averaging the result, one gets within zeros approximation $(w=v=0$ in Eqs.(11)) [19]

$$
C_{\nu \mathbf{k}}^{(0)}\left(\theta, \theta^{\prime}\right)=\frac{1+\left(r_{\mathbf{k}}-\mathrm{i} \nu\right) \theta+\left(r_{\mathbf{k}}+\mathrm{i} \nu\right) \theta^{\prime}}{r_{\mathbf{k}}^{2}+\nu^{2}} .
$$


Here, conventional frequency $\nu$ denotes Fourier transformation over "time" being the monomer number $n$. The most important feature of expression (20) is the characteric structure with respect to combination of the nilpotent variables $\theta, \theta^{\prime}$, that is inherent not only zeros approximation but arbitrary SUSY correlator. To this end it is convenient to introduce basis supervectors

$$
A\left(\theta, \theta^{\prime}\right)=\theta, \quad B\left(\theta, \theta^{\prime}\right)=\theta^{\prime}, \quad T\left(\theta, \theta^{\prime}\right)=1,
$$

containing functional product $X\left(\theta, \theta^{\prime}\right)=\int Y\left(\theta, \theta^{\prime \prime}\right) Z\left(\theta^{\prime \prime}, \theta\right) \mathrm{d} \theta^{\prime \prime}$ for any SUSY vectors $\mathbf{X}, \mathbf{Y}$, $\mathbf{Z}$. It is easy to see, that basis SUSY vectors (21) obey the following multiplication rules: $\mathbf{A}^{2}=\mathbf{A}, \mathbf{B}^{2}=\mathbf{B}, \mathbf{B T}=\mathbf{T}, \mathbf{T A}=\mathbf{T}$, other products are zero. Since the set of vectors $\mathbf{A}$, $\mathbf{B}, \mathbf{T}$ is closed, it is convenient to expand any SUSY correlator over this basis:

$$
\mathbf{C}=G_{+} \mathbf{A}+G_{-} \mathbf{B}+S \mathbf{T} .
$$

Hereafter the subscripts $\mathbf{k}, \nu$ are suppressed for brevity. Using Eqs.(16), (19), one gets for coefficients of expansion (22): $G_{+}=\left\langle\varphi^{*} \eta\right\rangle, G_{-}=\left\langle\eta^{*} \varphi\right\rangle, S=\left\langle|\eta|^{2}\right\rangle$. So, $G_{ \pm}$represent advanced and retarded Green functions and $S$ is the structure factor. In accordance with Eqs.(20), (21), (22), within zeroes approximation these functions are

$$
G_{ \pm}^{(0)}=(r \pm \mathrm{i} \nu)^{-1}, \quad S^{(0)}=G_{+}^{(0)} G_{-}^{(0)}=\left(r^{2}+\nu^{2}\right)^{-1} .
$$

The Dyson equation for the SUSY correlator (19) reads [12]

$$
\mathbf{C}^{-1}=\left(\mathbf{C}^{(0)}\right)^{-1}-w \mathbf{C}-\mathbf{\Sigma},
$$

where $w=2 \cdot 6^{1 / 3} \pi^{-4 / 3} D^{-1}(\sigma / l)^{2}$ is the typical value of the sum $\sum_{\mathbf{k}^{\prime}} w_{\mathbf{k k}^{\prime}}$ in Hamiltonian (11), $\Sigma$ is the SUSY self-energy operator. Using Eqs.(23) and expansions (22),

$$
\Sigma=\Sigma_{+} \mathbf{A}+\Sigma_{-} \mathbf{B}+\Sigma \mathbf{T},
$$

the Dyson equations for the components $G_{ \pm}, S$ become

$$
\begin{gathered}
G_{ \pm}^{-1}+w G_{ \pm}=(r \pm \mathrm{i} \nu)-\Sigma_{ \pm} \\
S=\left(1+2 \pi C_{2} l^{-1} \delta(\nu)+\Sigma\right) G_{+} G_{-}\left(1-w G_{+} G_{-}\right)^{-1} .
\end{gathered}
$$

Here $\delta$-term appears to take into account the contribution to Lagrangian due to quenched disorder. Coefficients of SUSY expansion (25) are [19]

$$
\begin{gathered}
\Sigma_{ \pm}(n)=\left(\mu^{2}+\frac{\lambda^{2}}{2} S(n)\right) S(n) G_{ \pm}(n) \\
\Sigma(n)=\left(\mu^{2}+\frac{\lambda^{2}}{6} S(n)\right) S^{2}(n)
\end{gathered}
$$

where the $\mathbf{r}-$ representation is used for macroscopically homogeneous system. 
Let us introduce now the memory parameter $q \equiv\langle\eta(n=N) \eta(n=0)\rangle$ and the nonergodicity parameter $\Delta \equiv g_{0}-g$ that is the difference between isothermal susceptibility $g_{0} \equiv$ $G_{-}(\nu=0)$ and thermodynamic value $g \equiv G_{-}(\nu \rightarrow 0)$. Being coefficients in SUSY expansion (22), the main correlators of non-ergodic system with memory acquire the elongated form:

$$
G_{ \pm}(\nu)=\Delta+G_{ \pm 0}(\nu), \quad S(n)=q+S_{0}(n)
$$

where the index 0 denotes the components corresponding to ergodic system without memory. Inserting Eqs.(28) to Eqs.(27) provides the self-energy components $\Sigma_{ \pm}(n), \Sigma(n)$ as functions of parameters $\Delta, q$ within the "time"-representation. Since the Dyson equations (26) require "frequency" Fourier transforms, it is convenient to use the fluctuation-dissipation theorem $S_{0}(n \rightarrow 0)=G_{ \pm 0}(\nu \rightarrow 0) \equiv g, \Sigma_{ \pm 0}(\nu \rightarrow 0)=\Sigma_{0}(n \rightarrow 0)$. As a result, the Dyson equation (26) gives in the $\nu$-representation

$$
\begin{gathered}
q\left[1-w g_{0}^{2}-\frac{1}{2}\left(\mu^{2}+\frac{\lambda^{2}}{3} q\right) q g_{0}^{2}\right]=C_{2} l^{-1} g_{0}^{2}, \\
S_{0}=\frac{\left(1+\Sigma_{0}\right) G_{+} G_{-}}{1-\left[w+\left(\mu^{2}+\lambda^{2} q / 2\right) q\right] G_{+} G_{-}} .
\end{gathered}
$$

The first of these equations corresponds to the $\delta$-term due to memory effects, the second one corresponds to "frequency" $\nu \neq 0$. At $\nu \rightarrow 0$ the characteristic product is $G_{+} G_{-} \rightarrow g^{2}$, so that the pole of the structure factor (29a)

$$
w+\left(\mu^{2}+\frac{\lambda^{2}}{2} q\right) q=g_{0}^{-2}
$$

determines the point of ergodicity breaking. Analogously, Eq.(26a) gives the equation for the susceptibility $g \equiv G_{-}(\nu \rightarrow 0)$

$$
w g^{2}+\frac{\mu^{2}}{2} g\left[(g+q)^{2}-q^{2}\right]+\frac{\lambda^{2}}{6} g\left[(g+q)^{3}-q^{3}\right]=r g-1 .
$$

The system of equations (29)-(31) completely describes thermodynamic behaviour of random heteropolymer by analogy with the spin glass theory [7]. So, Eqs.(29), (31) play a role of Sherrington-Kirkpatrick equations, and Eq.(30) defines the point of de Almeida-Thouless instability.

According to Eqs.(29), (30), the memory parameter is given by the cubic equation

$$
\left(\mu^{2} / 2+\lambda^{2} q / 3\right) q^{2}=C_{2} l^{-1} .
$$

Taking into account definitions (11), it is seen that for copolymers close to symmetric composition $f=0.5\left(C_{3} \ll C_{2}\right)$ the first term in brackets of Eq.(32) is negligible, and the dependence $q \propto l^{1 / 3}$ takes place. In the opposite case of dilute copolymer, where $f \ll 1$ $\left(C_{2} \ll C_{3}\right)$, we have the less value $q \propto f l^{1 / 2}$. Naturally, the memory parameter diminishes always with correlation length decreasing.

The behaviour of isothermal, $g_{0}$, and thermodynamic, $g$, susceptibilities is described by the equations (30) and (31), respectively. The magnitude $g_{0}$ depends only on the quenched 
disorder $l$, whereas the latter, $g$, is defined by both values $l, \chi$ of quenched and thermal disorders (see Eqs.(11) for dependence $r(\chi)$ ). The point of ergodicity breaking, $r_{0}$, is determined by equation

$$
2-r g_{0}+\frac{\mu^{2}}{2} g_{0}^{3}+\frac{\lambda^{2}}{6} g_{0}^{3}\left(g_{0}+3 q\right)=0,
$$

that follows from Eqs.(30), (31) at $g=g_{0}$. At the point of MS, $r_{c}$, one has $\mathrm{d} g / \mathrm{d} r=-\infty$, and Eq.(31) gives the condition

$$
w+\mu^{2}(g+q)+\frac{\lambda^{2}}{2}(g+q)^{2}=g^{-2} .
$$

The form of phase diagram given by Eqs.(33), (34) is shown in Fig.1. 


\section{REFERENCES}

[1] C.D. Sfatos, E.I. Shakhnovich, Phys. Rep. 288, 77 (1997).

[2] L. Leibler, Macromolecules 13, 1602 (1980).

[3] G.H. Fredrickson, E. Helfand, J. Chem. Phys. 87, 697 (1987).

[4] V.S. Pande, A.Yu. Grosberg, T. Tanaka, Phys. Rev. E51, 3381 (1995).

[5] A.V. Dobrynin, I.Ya. Erukhimovich, J. Phys. I France 5, 365 (1995).

[6] S. Stepanov, A.V. Dobrynin, T.A. Vilgis, K. Binder, J. Phys. I France 6, 837 (1996).

[7] M. Mezard, G. Parisi, M.A. Virasoro, Spin Glass Theory and Beyond (World Scientific, Singapore, 1987).

[8] G.H. Fredrickson, S.T. Milner, L. Leibler, Macromolecules 25, 6341 (1992).

[9] E.G. Timoshenko, Yu.A. Kuznetsov, K.A. Dawson, Phys. Rev. E54, 4071 (1996).

[10] J. Zinn-Justin, Quantum Field Theory and Critical Phenomena (Clarendon Press, Oxford, 1993).

[11] J. Kurchan, J. Phys. I France 21333 (1992).

[12] A.I. Olemskoi, I.V. Koplyk, Physics-Uspekhi 38, 1061 (1995).

[13] T.A. Vilgis, J. Phys. A24, 5321 (1991).

[14] M. Doi, S.F. Edwards, The Theory of Polymer Dynamics (Clarendon Press, Oxford, 1986).

[15] T. Halpin-Healy, Y.-C. Zhang, Phys. Rep. 254, 215 (1995).

[16] A.I. Olemskoi, Uspekhi Fizicheskikh Nauk 168, 287 (1998).

[17] H. Risken, The Fokker-Planck Equation (Springer, Berlin-Heidelberg, 1989).

[18] R.J. Glauber, J. Math. Phys. 4, 294 (1963).

[19] A.I. Olemskoi, V.A. Brazhnyi, to be published. 


\section{FIGURES}

FIG. 1. The phase diagram for random copolymer with $\sigma=0$ (a) and $\sigma=5$ (b) ( $l=1, D=1$; solid and broken curves are the MS and ergodicity breaking lines correspondingly) 
This figure "fig.gif" is available in "gif" format from: http://arxiv.org/ps/cond-mat/9807063v1 